\title{
On the Set of All Stabilizing First-Order Controllers
}

\author{
K. Saadaoui and A. B. Özgüler \\ Department of Electrical and Electronics Engineering, \\ Bilkent University, Ankara, Turkey. \\ karim@ee.bilkent.edu.tr, ozguler@ee.bilkent.edu.tr.
}

\begin{abstract}
A computational method is given for determining the set of all stabilizing proper first-order controllers for finite dimensional, linear, time invariant, scalar plants. The method is based on a generalized Hermite-Biehler theorem.
\end{abstract}

Keywords: Hermite-Biehler theorem, stabilization, linear systems.

\section{Introduction}

In [1], a computational characterization of all stabilizing proportional-integral (PI) and proportionalintegral-derivative (PID) controllers was derived. In [2], an alternative fast method for determining all stabilizing PID controllers was derived. The limiting values of controller parameters that guarantee stability are determined in [1] using an extension of the HermiteBiehler theorem [3], and using the Nyquist plot in [2]. In this paper, we give an extension of the method of [1] to solve the problem of determining all first-order controllers that stabilizes a given plant. The paper is organized as follows. In section 2 , the method for calculating stabilizing gains is revisited. In section 3 , we give an algorithm for determining stabilizing first-order controllers. Finally, section 4 contains some concluding remarks.

\section{Proportional Controllers}

Let $\mathbf{C}$ denote the set of complex numbers and let $\mathbf{C}_{-}, \mathbf{C}_{0}, \mathbf{C}_{+}$denote the points in the open left half, $j \omega$-axis, and the open right half of the complex plane, respectively. Then, the set $\mathcal{H}$ of Hurwitz stable polynomials are $\mathcal{H}=\left\{\psi(s) \in \mathbf{R}[s]: \psi(s)=0 \Rightarrow s \in \mathbf{C}_{-}\right\}$. The derivative of $\psi$ is denoted by $\psi^{\prime}$ and the signature $\sigma(\psi)$ of a polynomial $\psi \in \mathbf{R}[s]$ is the difference between the number of its $\mathbf{C}_{-}$roots and $\mathbf{C}_{+}$roots. Given $\psi \in \mathbf{R}[s]$, the even-odd components $(a, b)$ of $\psi(s)$ are the unique polynomials $a, b \in \mathbf{R}[u]$ such that $\psi(s)=a\left(s^{2}\right)+s b\left(s^{2}\right)$. Finally, the greatest common divisor of $a$ and $b$ is denoted by $\operatorname{gcd}\{a, b\}$ and $\lfloor m\rfloor$ denotes the greatest integer less than or equal to $m \in \mathbf{R}$. We state the following result for later reference. The proof of this result follows by a generalization of the Hermite-Biehler theorem [3]
Lemma 1. A nonzero polynomial $\psi \in \mathbf{R}[s]$ has $r$ real negative roots without counting the multiplicities if and only if the signature of the polynomial $\psi\left(s^{2}\right)+s \psi^{\prime}\left(s^{2}\right)$ is $2 r$. All roots of $\psi$ are real, negative, and distinct if and only if $\psi\left(s^{2}\right)+s \psi^{\prime}\left(s^{2}\right) \in \mathcal{H}$.

We now describe a slight extension of the constant stabilizing gain algorithm of [3]. Given a plant $g(s)=\frac{p(s)}{q(s)}$, where $p, q \in \mathbf{R}[s]$ are nonzero with $m=\operatorname{deg} p$ less than or equal to $n=\operatorname{deg} q$, the set $A_{r}(p, q):=\{\alpha \in \mathbf{R}$ : $\sigma[\phi(s, \alpha)]=\sigma[q(s)+\alpha p(s)]=r, \operatorname{deg} \phi=\operatorname{deg} q\}$, is the set of all real $\alpha$ such that $\phi(s, \alpha)$ has signature equal to $r$. Let $(h, g)$ and $(f, e)$ be the even-odd components of $q$ and $p$, respectively. Let $d:=\operatorname{gcd}\{f, e\}$ so that $f=$ $d \bar{f}, e=d \bar{e}$, or coprime polynomials $\bar{f}, \bar{e} \in \mathbf{R}[u]$. Then, the polynomial $\bar{p}(s):=\bar{f}\left(s^{2}\right)+s \bar{e}\left(s^{2}\right)=p(s) / d\left(s^{2}\right)$ is free of $\mathbf{C}_{0}$ roots except possibly a simple root at $s=0$. Let $(H, G)$ be the even-odd components of $q(s) \bar{p}(-s)$. Also let $F\left(s^{2}\right):=p(s) \bar{p}(-s)$. By a simple computation, it follows that $H(u)=h(u) \bar{f}(u)-u g(u) \bar{e}(u), G(u)=$ $g(u) \bar{f}(u)-h(u) \bar{e}(u)$, and $F(u)=f(u) \bar{f}(u)-u e(u) \bar{e}(u)$. If $G \not \equiv 0$ and if they exist, let the real negative zeros with odd multiplicities of $G(u)$ be $\left\{v_{1}, \ldots, v_{k}\right\}$ with the ordering $v_{1}>v_{2}>\cdots>v_{k}$, with $v_{0}:=0$ and $v_{k+1}:=-\infty$ for notational convenience. The following algorithm determines whether $A_{r}(p, q)$ is empty or not and outputs its elements when it is not empty:

Algorithm 1.

1. Calculate $\alpha_{i}= \begin{cases}-\frac{H}{F}\left(v_{i}\right), i=0, \ldots, k & \text { for odd } r-m \\ -\frac{H}{F}\left(v_{i}\right), i=0, \ldots, k+1 & \text { for even } r-m,\end{cases}$

and sort them in ascending order $\bar{\alpha}_{0}<\bar{\alpha}_{1}<\ldots<$ $\bar{\alpha}_{k+2}<\bar{\alpha}_{k+3}$ where $\bar{\alpha}_{0}=-\infty$ and $\bar{\alpha}_{k+3}=\infty$.

2. Identify all the sequences of signums

$$
\mathcal{I}= \begin{cases}\left\{i_{0}, i_{1}, \ldots, i_{k}\right\} & \text { for odd } r-m \\ \left\{i_{0}, i_{1}, \ldots, i_{k+1}\right\} & \text { for even } r-m\end{cases}
$$

where $i_{0} \in\{-1,0,1\}$ and $i_{j} \in\{-1,1\}$ for $j=$ $1, \ldots, k+1$, that correspond to the intervals $\left(\bar{\alpha}_{i}, \bar{\alpha}_{i+1}\right)$ for $i=0, \ldots, k+2$.

3. For each signum sequence $\mathcal{I}_{i}$ from step 2 , if

$$
\begin{aligned}
& r-\sigma(p)= \begin{cases}i_{0}-2 i_{1}+\cdots+2(-1)^{k} i_{k} & r-m \text { odd } \\
i_{0}-\cdots+(-1)^{k+1} i_{k+1} & r-m \text { even. }\end{cases} \\
& \text { holds, then }\left(\bar{\alpha}_{i}, \bar{\alpha}_{i+1}\right) \in A_{r}(p, q)
\end{aligned}
$$

Remark 1. By step 3 of Algorithm 1, a necessary condition for the existence of an $\alpha \in A_{r}(p, q)$ is 
that the odd part of $[q(s)+\alpha p(s)] \bar{p}(-s)$ has at least $\bar{r}=\max \left\{0,\left\lfloor\frac{|r-\sigma(p)|-1}{2}\right\rfloor\right\}$ real negative roots with odd multiplicities. When solving a constant stabilization problem this lower bound is $\bar{r}=\max \left\{0,\left\lfloor\frac{\mid n-\sigma(p)\rfloor-1}{2}\right\rfloor\right\}$.

\section{First-Order Controllers}

A first-order controller $c(s)=\frac{\alpha_{2} s+\alpha_{3}}{s+\alpha_{1}}$ applied to $g_{0}(s)=\frac{p_{0}(s)}{q_{0}(s)}$, with $m=\operatorname{deg} p_{0}$ less than or equal to $n=\operatorname{deg} q_{0}$, gives the closed loop characteristic polynomial

$$
\begin{aligned}
\phi_{1}\left(s, \alpha_{1}, \alpha_{2}, \alpha_{3}\right) & =\left(s+\alpha_{1}\right) q_{0}(s)+\left(\alpha_{2} s+\alpha_{3}\right) p_{0}(s) \\
& =q_{1}(s)+\alpha_{3} p_{1}(s)
\end{aligned}
$$

Multiplying $\phi_{1}\left(s, \alpha_{1}, \alpha_{2} ; \alpha_{3}\right)$ by $\bar{p}_{1}(-s)$ we obtain

$$
\begin{aligned}
\psi_{1}\left(s, \alpha_{1}, \alpha_{2}, \alpha_{3}\right)= & \phi_{1}\left(s, \alpha_{1}, \alpha_{2}, \alpha_{3}\right) \bar{p}_{1}(-s) \\
= & s^{2} G\left(s^{2}\right)+\alpha_{1} H\left(s^{2}\right)+\alpha_{3} F\left(s^{2}\right) \\
& +s\left[H\left(s^{2}\right)+\alpha_{1} G\left(s^{2}\right)+\alpha_{2} F\left(s^{2}\right)\right]
\end{aligned}
$$

Note that $\alpha_{1}, \alpha_{2}$ appear in the odd part and $\alpha_{1}, \alpha_{3}$ appear in the even part. It is no longer possible to exploit the results given in [1] and proceed.

The reasoning behind the below algorithm can be explained as follows. Suppose that $\phi_{1}(s)$ is Hurwitz stable for some $\alpha_{1}, \alpha_{2}, \alpha_{3} \in \mathbf{R}$. By Remark 1, it follows that the odd part of $\psi_{1}(s)$ has at least $r_{1}=\left\lfloor\frac{n-\sigma\left(p_{1}\right)}{2}\right\rfloor$ real negative roots with odd multiplicities. By Lemma 1 , $\sigma\left[\phi_{2}(s)\right]=2 r_{1}$, where

$$
\begin{aligned}
\phi_{2}(s)= & H\left(s^{2}\right)+\alpha_{1} G\left(s^{2}\right)+\alpha_{2} F\left(s^{2}\right) \\
& +s\left[H^{\prime}\left(s^{2}\right)+\alpha_{1} G^{\prime}\left(s^{2}\right)+\alpha_{2} F^{\prime}\left(s^{2}\right)\right] \\
= & q_{2}(s)+\alpha_{2} p_{2}(s)
\end{aligned}
$$

In order to find the suitable ranges of $\alpha_{1}$ and $\alpha_{2}$, we modify $\phi_{2}(s)$ as follows.. Let $B:=\operatorname{gcd}\left\{F, F^{\prime}\right\}$ so that $F=B \vec{F}, F^{\prime}=B \bar{F}^{\prime}$ for coprime polynomials $\vec{F}, \vec{F}^{\prime} \in \mathbf{R}[u]$. Let $p_{2}(s):=\bar{F}\left(s^{2}\right)+s \bar{F}^{\prime}\left(s^{2}\right)$. Then, by a straightforward computation, $\psi_{2}(s)=\phi_{2}(s) \bar{p}_{2}(-s)=$ $I\left(s^{2}\right)+\alpha_{1} J\left(s^{2}\right)+\alpha_{2} K\left(s^{2}\right)+s\left[L\left(s^{2}\right)+\alpha_{1} M\left(s^{2}\right)\right]$, where $I(u), J(u), K(u), L(u)$, and $M(u)$ can be easily computed. Once more by Remark 1 , the odd part of $\psi_{2}(s)$ has at least $r_{2}=\left\lfloor\frac{\left|2 r_{1}-\sigma\left(p_{2}\right)\right|-1}{2}\right\rfloor$ real negative roots with odd multiplicities. Values of $\alpha_{1} \in \mathbf{R}$ achieving $r_{2}$ real negative roots with odd multiplicities can be determined using Lemma 1 and Algorithm 1. In Algorithm 2 below, we follow a nested procedure where one $\alpha_{1}$, $\alpha_{2}$ is determined and then the corresponding values of $\alpha_{3}$ are obtained.

\section{Algorithm 2.}

1. Using Lemma 1 and Algorithm 1, partition the real axis into intervals such that in every interval $L(u)+$ $\alpha_{1} M(u)$ has a constant number of real negative roots with odd multiplicities.

2. Fix $r_{1}=\left\lfloor\frac{n-\sigma\left(p_{1}\right)}{2}\right\rfloor$.

(a) Determine from step 1 if a range of $\alpha_{1}$ for which $r_{2}=\left\lfloor\frac{\left\lfloor 2 r_{3}-\sigma\left(p_{2}\right)\right\rfloor-1}{2}\right\rfloor$ exists. i. Fix an $\alpha_{1}$ in the range of step 2.a.

ii. Apply Algorithm 1 to $q_{2}(s)$ replacing $q(s)$ , $p_{2}(s)$ replacing $p(s)$, and $\sigma\left(\phi_{2}\right)=2 r_{1} r e-$ placing $r$. (This calculates admissible values of $\alpha_{2}$ such that $H(u)+\alpha_{1} G(u)+\alpha_{2} F(u)$ has $r_{1}$ real negative roots with odd multiplicities.)

A. Fix an $\alpha_{2}$ from the range determined in 2.a.ii.

B. Apply Algorithm 1 to $q_{1}(s)$ and $p_{1}(s)=$ $p(s)$. (This calculates all admissible values of $\alpha_{3}$ such that $\phi_{1}$ of (1) is in H.)

C. Increment $\alpha_{2}$ and go to step 2.a.ii.A. iii. Increment $\alpha_{1}$ and go to step 2.a.i.

(b) Increment $r_{1}$ by one, if $r_{1} \leq \operatorname{deg}(H(u)+$ $\left.\alpha_{1} G(u)+\alpha_{2} F(u)\right)$ go to step 2.a.

Example 1. Consider determining proper first-order controllers to stabilize the plant $g_{0}(s)=\frac{p_{0}(s)}{q_{0}(s)}$ given in $[1]$, where $q_{0}(s)=s^{5}+3 s^{4}+29 s^{3}+15 s^{2}-3 s+60$ and $p_{0}(s)=s^{3}-6 s^{2}+2 s+1$. Algorithm 2 outputs the set of stabilizing $\left(\alpha_{1}, \alpha_{2}, \alpha_{3}\right)$ values as shown in Figure 1.

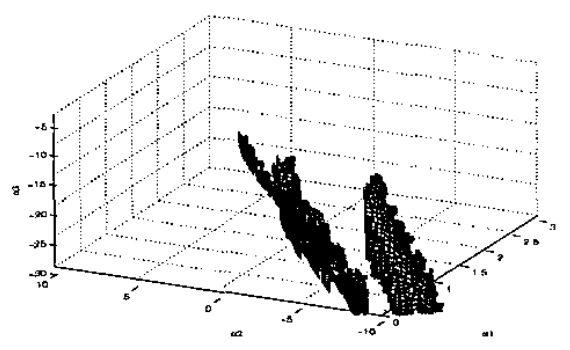

Figure 1: Stabilizing set of $\left(\alpha_{1}, \alpha_{2}, \alpha_{3}\right)$ values.

\section{Conclusions}

In this paper a solution is given to the problem of determining all first-order controllers that stabilize a given plant. The method consists of an application of constant stabilizing gain characterization on two auxiliary plants. An extension of this method to any fixed order controller is reported in [5] and to interval plants is reported in [4].

\section{References}

[1] Datta, A., Ho, M. T. and S. P. Bhattacharyya, Structure and Synthesis of PID controllers, New York: Springer-Verlag, 2000.

[2] Munro, N. and M. T. Söylemez, "Fast computation of stabilizing PID controllers for uncertain parameter systems", Proc. $3^{\text {rd }}$ IFAC ROCOND, Czech Republic, 2000.

[3] Özgüler, A. B. and A. A. Koçan, "An analytic determination of stabilizing feedback gains", Report, Institut für Dynamische Systeme, Report no. 321, Universität Bremen, 1994.

[4] Saadaoui, k. and A. B. Özgüler, "Computation of stabilizing first-order controllers for interval plants", Proc. $2^{\text {nd }}$ International Conference on Signals, Systems, Decision and Information Technology, Tunisia, 2003.

[5] Saadaoui, k. and A. B. Özgüler, "A new method for the computation of all stabilizing controllers of a given order", Technical Report, Bilkent University, 2003. 